\title{
Determination of an infectious dose of Leptospira for the performance of challenge test in assessing the efficacy of Leptospira vaccines
}

\author{
Jiří Nepeřený, Josef Chumela, Vladimír Vrzal \\ Bioveta, a.s., Ivanovice na Hané, Czech Republic
}

Received August 9, 2010

Accepted September 13, 2011

\begin{abstract}
The efficacy of vaccines against leptospiral disease can be determined objectively by challenge test in experimental animals. Selection of suitable leptospiral challenge strains and determination of an optimal challenge dose to prove exactly that the given vaccine Leptospira serotype induces protective immunity in vaccinated dogs is a critical point in performing challenge experiments. The aim of our study was to verify and determine an appropriate challenge dose for efficacy tests in dogs for the following Leptospira serovars: L. grippotyphosa, L. icterohaemorrhagiae and L. canicola. The appropriate challenge dose was determined on the basis of pathognomonic symptoms of infection, Leptospira capture at cultivation and pathological changes in dogs infected experimentally with various doses $\left(5 \times 10^{4}-5 \times 10^{8}\right)$ of Leptospira serovars. A dose of $5 \times 10^{6}$ of each respective serovar administered intraperitoneally was determined to be a suitable challenge dose. The dogs infected with the selected dose showed the typical symptoms of the disease and met the requirements of an objective and standard evaluation of the vaccine efficacy according to the pharmacopoeial monograph. A study of such extent was done for the first time in the Czech Republic.
\end{abstract}

Vaccine against leptospirosis, determination of efficacy, challenge test, determination of infectious dose

Leptospirosis is an infectious disease of animals and humans caused by spirochaetes of the genus Leptospira. It is a worldwide zoonotic infection with a much greater incidence in tropical regions and has now been identified as one of the emerging infectious diseases (Vinetz 2001; Levet 2001; Roca 2006; Vijayachari et al. 2008). The bacteria cause a polyfactorial disease from asymptomatic infection, subclinical cases, up to acute infections with a fatal outcome. One of the ways of protecting animals from leptospirosis is vaccination, which may reduce the incidence and severity of the disease. Currently, not only proven whole-cell inactivated vaccines are used, but also new vaccines are being tested: recombinant vaccines based on outer membrane proteins (OMP), lipopolysaccharide vaccines, DNA vaccines, etc. (Srivastava 2006; Wang et al. 2007).

To increase the immune response, a mineral adjuvant (aluminum hydroxide) is mainly used. Regarding the fact that Leptospira is a weak immunogen, vaccinations with a subsequent revaccination are used at the start of immunoprophylaxis to create an antibody content. Sufficient titre of antibodies is then maintained by regular annual revaccination. The efficacy of Leptospira vaccines was formerly tested in hamsters. The laboratory hamster (Mesocricetus auratus) is regarded a very sensitive and suitable model animal for testing of Leptospira infections and has been used for a long time (Randall and Cooper 1944). Using of hamsters in testing of efficacy of Leptospira vaccines was mentioned in earlier editions of the pharmacopoeia. The efficacy test using the challenge in dogs appeared in the monograph of Czech Pharmacopoeia (2005), which corresponds to the fifth edition of the European Pharmacopoeia (EP). The preceding pharmacopoeia ĆL 2002 - monograph "Vaccinum leptospirosis ad usum veterinarium", described the challenge test only in hamsters.

Currently, Leptospira canicola, L. icterohaemorrhagiae, L. sejroe, L. grippotyphosa, L. bratislava and L. pomona are the most important serovars that cause infections in dogs

Address for correspondence:

MVDr. Jiří Nepeřený

Bioveta, a.s., Komenského 212

68323 Ivanovice na Hané, Czech Republic

Phone: +420 517318553

E-mail: nepereny.jiri@bioveta.cz

http://www.vfu.cz/acta-vet/actavet.htm 
(Boutilier et al. 2003; Schreiber et al. 2005). An efficient way of preventing leptospirosis in dogs is immunoprophylaxis with effective, especially multivalent vaccines (Klaasen et al. 2003; Schreiber et al. 2005). Protective efficacy against each Leptospira serovar contained in the vaccine must be demonstrated by a challenge test in dogs before registering and using these immunobiological preparations in practice (European Pharmacopoeia, Article 0447). The critical point in performing of challenge experiments is to determine optimal challenge doses of individual Leptospira serovars so as to demonstrate exactly that the given vaccine Leptospira serotype induces protective immunity in vaccinated dogs. The maintenance of challenge Leptospira strains for testing the efficacy of vaccines against canine leptospirosis is described by Reed et al. (2000).

The performance and results of challenge tests in dogs have been described recently in a series of papers (Greenlee et al. 2004; Schreiber et al. 2005a; Schreiber et al. 2005b; Greenlee et al. 2005). Most authors evaluate the efficacy of the vaccine Leptospira component serologically, mainly using the agglutination reaction - lysis (MAL), when specific agglutination-lytic antibodies are determined in vaccinated animals. However, only one part of the immune response is assessed like this. It has been proved experimentally that the cell-mediated immunity (CMI) plays an important role at the onset of immunity and in the protection against leptospirosis (Koizumi and Watanabe 2005).

During the development of inactivated veterinary vaccines against canine leptospirosis it is important to ensure that the registration dossier includes all the required validations and testing as required, in particular: Special requirements for veterinary immunobiological products (Directive 92/18/EEC/1992) in the European Union, any new product shall comply with the European Pharmacopoeia monograph and its supplements issued by the Council of Europe, any new product shall meet the recommendations of international organizations (WHO, OIE), any new product shall meet specific and additional requirements of the state where it is registered. The aim of our study was to determine the optimal challenge dose of leptospira strains for the efficacy testing of vaccine against leptospirosis in dogs using challenge test in accordance with the guidelines of the European Pharmacopoeia monograph 01/2005:0447.

\section{Materials and Methods}

Fifteen eight-week-old female Beagle dogs, without detectable antibodies against L. grippotyphosa, L. icterohaemorrhagiae and L. canicola by microscopic agglutination test (MAL), from a commercial breed of laboratory animals (Biotest Konárovice, Czech Republic) were used in this study. Animals were randomly divided into 3 groups of 5 dogs. One group received the serovar L. grippotyphosa, the second group received the serovar L. icterohaemorrhagiae and the third group received the serovar L. canicola.

Challenge strains of Leptospira used in the test must be different from the strains used for vaccine production. The strains L. grippotyphosa, L. icterohaemorrhagiae and L. canicola were acquired from the American Type Culture Collection (ATCC) via LGC Promochem (United Kingdom). In the selection of strains, emphasis was laid to their pathogenicity. All Leptospira strains were kept in liquid nitrogen at $-196{ }^{\circ} \mathrm{C}$ on a long-term basis. The selected Leptospira strains were cultured in liquid culture medium Stuart with an addition of $10 \%$ rabbit serum SIGMA (R4505) at $28^{\circ} \mathrm{C}$. The number of Leptospirae in the culture was counted by means of Petroff Hausser counting chamber (Electron Microscopy Sciences, cat. 63512-21) in a dark field of microscope. The concentration of Leptospira of each serovar was adjusted by dilution with sterile saline so that $1 \mathrm{ml}$ of culture contained $10^{4}, 10^{5}, 10^{6}, 10^{7}$ and $10^{8}$ Leptospirae. The infectious doses of individual Leptospira strains used for infection in dogs are described in table No. 1

An infectious dose for each animal was $5.0 \mathrm{ml}$ and was administered intraperitoneally after a seven-day acclimatization period. All animals were observed for 28 days. During the whole experiment, body temperature and clinical status of experimental animals were recorded daily. Body weight of animals was recorded before and after the experiment.

The following pathognomonic signs of leptospirosis infection were selected as critical for determining an appropriate challenge dose of Leptospira: 1) Culture detection of Leptospira in the blood before the trial and on days 2, 3, 4, 5, 8 and 11 post infection by microscopic evaluation, 2) Culture detection of Leptospira in the urine before the trial and on days $3,5,8,11,14,21$ and 28 post infection by microscopic evaluation, 3) Culture detection of Leptospira in the kidney, liver and gall bladder by microscopic evaluation at dissection on day 28 post infection or on the day of death, 4) Clinical symptoms of leptospirosis during the test period in particular: 
Table 1. Infectious doses of individual Leptospira strains

\begin{tabular}{cccccc}
\hline \multicolumn{2}{c}{$\begin{array}{c}\text { L. grippotyphosa } \\
\text { Dog no. }\end{array}$} & Leptospirae in a dose & Dog no. & Leptospirae in a dose & Dog no. Leptospirae in a dose \\
\hline 1 & $5 \times 10^{4}$ & 6 & $5 \times 10^{4}$ & 11 & $5 \times 10^{4}$ \\
2 & $5 \times 10^{5}$ & 7 & $5 \times 10^{5}$ & 12 & $5 \times 10^{5}$ \\
3 & $5 \times 10^{6}$ & 8 & $5 \times 10^{6}$ & 13 & $5 \times 10^{6}$ \\
4 & $5 \times 10^{7}$ & 9 & $5 \times 10^{7}$ & 14 & $5 \times 10^{7}$ \\
5 & $5 \times 10^{8}$ & 10 & $5 \times 10^{8}$ & 15 & $5 \times 10^{8}$ \\
\hline
\end{tabular}

icterus of mucous membranes (conjunctiva and oral mucosa) and skin (abdomen and genitals), conjuctivitis, anorexia, weight loss, overall symptoms of the disease (apathy, untidy hair, fearfulness), 5) Dissection - icterus of serosae (peritoneum, pleura, intestinal serosae), enlarged or fragile liver, enlarged spleen and enlarged gall bladder.

The study was approved by the Ethics Committee of Bioveta plc Ivanovice na Hané and was carried out in accordance with the Act on Animal Health and Animal Welfare of Czech Republic.

\section{Results}

L. grippotyphosa group:

In dog no. 1 (infectious dose of $5 \times 10^{4}$ ), Leptospira was isolated from the blood on day 4 after infection and from the kidney. No clinical symptoms of disease or pathological changes were observed.

In dog no. 2 (infectious dose of $5 \times 10^{5}$ ), Leptospira was isolated from the blood on day 4, 5 and 11 post infection (p. i.) and from the urine sample taken on day $21 \mathrm{p}$. i. This dog manifested conjunctivitis and mucous membrane icterus. No changes were observed at dissection.

In dog no. 3 (infectious dose of $5 \times 10^{6}$ ), Leptospira was isolated from the blood samples on days 4 and $5 \mathrm{p}$. i and from the urine sample taken on day $21 \mathrm{p}$. i. This dog manifested conjunctivitis and mucous membrane icterus. Enlargement of the liver and spleen and icteric discoloration of serosae were detected at dissection. During the observation period the dog was suffering from anorexia and weight loss.

Dog no. 4 (infectious dose of $5 \times 10^{7}$ ), died on day 19 p. i. Leptospira was isolated from a blood sample on day 5 p. i. and from the urine, liver, kidney and gall bladder taken on the day of death. Post-mortem examination on the day of death detected marked icterus of serosae, enlarged liver, spleen and gall bladder. Consistency of the liver tissue was very fragile.

Dog no. 5 (infectious dose of $5 \times 10^{8}$ ) died on day 15 p. i. Leptospira was isolated from the blood on days 4 and 8 p. i. and from the urine samples collected on day 5 p. i. and on the day of death. Isolation was also successful from a sample of liver and gall bladder; isolation from the kidney was negative because of bacterial contamination of the sample. Pathological changes were consistent with the changes found in dog no. 4.

L. icterohaemorrhagiae group:

In dog no. 6 (infectious dose of $5 \times 10^{4}$ ) and dog no. 7 (infectious dose of $5 \times 10^{5}$ ) Leptospira was not isolated from any sample of biological material. No clinical symptoms of disease or pathological changes were observed.

In dog no. 8 (infectious dose of $5 \times 10^{6}$ ), Leptospira was isolated from a blood sample on day 5 p.i. and from the urine sample taken on day 21 p.i. This dog also manifested conjunctivitis and mucous membrane icterus. Enlargement of the liver and spleen was detected at dissection.

In dog no. 9 (infectious dose of $5 \times 10^{7}$ ), Leptospira was isolated from the blood samples taken on days 4 and 8 p.i. and from the urine samples taken on days 14 and 
21 p.i. Post-mortem examination found icterus of serosae and enlargement of the liver and spleen. During the observation period the dog was suffering from anorexia and lost weight.

In dog no. 10 (infectious dose of $5 \times 10^{8}$ ), Leptospira was isolated from the blood samples taken on days 4 and 5 p.i. and from the urine samples taken on days 11 and 14 p.i. Isolation from the kidney sample taken during the postmortem examination on day 28 p.i. was also positive. The post-mortem examination revealed marked icterus of serosae, enlarged liver, spleen and gall bladder. The consistency of the liver tissue was very fragile. During the observation period the dog was suffering from anorexia and lost weight.

\section{L. canicola group:}

In dog no. 11 (infectious dose of $5 \times 10^{4}$ ) and dog no. 12 (infectious dose of $5 \times 10^{5}$ ), Leptospira was isolated from the blood samples taken on days 2 and 8 p.i. Clinical or pathological changes were not observed.

In dog no. 13 (infectious dose of $5 \times 10^{6}$ ), Leptospira was isolated from the blood samples on days 2 and 8 p.i. and from the urine sample taken on day 21 p.i. Leptospira was also isolated from the samples of kidney, liver and gall bladder taken at necropsy. This dog also manifested conjunctivitis and mucous membrane icterus. Enlargement of the liver and spleen and icterus of serosae were detected at necropsy. The dog was suffering from anorexia and lost weight.

In dog no. 14 (infectious dose of $5 \times 10^{7}$ ), Leptospira was isolated from the blood sample taken on day 8 and from the urine samples taken on days 3 and 14 p.i. and from the kidney. The sample taken from the gall bladder was contaminated.

In dog no. 15 (infectious dose of $5 \times 10^{8}$ ), Leptospira was isolated from the blood samples taken on days 2 and 8 p.i. and from the urine sample taken on day 14 p.i. Isolation of the kidney sample was also positive, a sample of the gall bladder could not be assessed due to bacterial contamination. Clinical symptoms of the disease (mucosal icterus and conjunctivits) and pathological changes (pronounced icterus of serosae, enlarged liver, spleen and gall bladder and fragile consistency of the liver tissue) were identical in dogs no. 14 and 15. Both animals also suffered from anorexia and weight loss.

\section{Discussion}

The aim of this study was to verify and determine an appropriate challenge dose for efficacy tests in dogs for the following Leptospira serovars: Leptospira grippotyphosa, $L$. icterohaemorrhagiae and L. canicola.

Determination of the challenge dose is a central point of successful realisation of the challenge test in evaluation of the leptospiral vaccines efficacy. The accurate conditions of challenge dose are not specified in the pharmacopoeial monograph. They depend on many factors and for each strain used in test are strictly individual. The pathogenicity of the Leptospira strain depends primarily on the number of their passages. Repeated passaging of Leptospira serovars in susceptible hosts can increase the pathogenicity of strains (Haake 2006). Similar tests have been performed abroad, but different Leptospira strains and different laboratory animals were used, especially hamsters (Silva et al. 2008). According to EP monograph, the evaluation of efficacy of leptospiral vaccines has to be performed using challenge test on the target animals.

In our study, we performed challenge experiments in the dog model with three highly virulent Leptospira strains. The challenge dose $5 \times 10^{6}$ which was determined in our test after intraperitoneally application to dogs proved to be the best for the achieving the symptoms of infection. This dose used for experimental infection has shown and met the evaluation criteria of the EP monograph (culture detection of Leptospira in the blood and urine, clinical symptoms and pathological changes). The dose has to be strong enough to 
induce disease in unvaccinated animals, but not so strong enough to cause breaking of the immunity in vaccinated animals in challenge test.

The efficacy assessment of the leptospiral vaccines using challenge test has been described several times. The higher doses $\left(10^{8}-10^{9}\right)$ were used for experimental infection of dogs, because different Leptospira strains were used (Klaasen et al 2003; Schreiber et al. 2005).

The dose of Leptospira strains used for the challenge tests, not for the induction of infection, is a strictly individual matter. The appropriate challenge dose determination has to be done before each new challenge test.

In our work, which has been performed for the first time in the Czech Republic in this range, we described a procedure for the determination of the appropriate challenge dose of Leptospira for the objective assessment of the vaccine against leptospirosis efficacy, containing serovars L. grippotyphosa, L. canicola and L. icterohaemorrhagiae. The selected dose cannot be compared with the doses used in similar tests in other countries, since other authors have used other strains, or they have performed these tests using the other species of the laboratory animals. Larger animals, such as dogs, may be more appropriate for the assessment study of renal physiology. Moreover, vaccines must also be verified in the target animal (Haake 2006). The infectious dose was chosen so that it always produced an evaluable response in control dogs (detection from the blood, urine, clinical symptoms, pathological changes), but no death in a control non-vaccinated dogs. Calculation of a challenge dose by Reed-Muench (Reed and Muench 1938) cannot be used.

An optimal challenge dose $5 \times 10^{6}$ of each tested Leptospira serovar, i.e. L. grippotyphosa, L. icterohaemorrhagiae and L. canicola was determined as suitable to determine the efficacy of vaccines against canine leptospirosis according to the European Pharmacopoeia Article 0447. With this challenge dose it can be evaluated if the tested vaccine produces protective immunity against clinical manifestations of disease, against infection or urinary tract infection and excretion of Leptospira.

\section{References}

Boutilier P, Carr A, Shulman RL 2003: Leptospirosis in dogs: A serological survey and case series 1996 to 2001. Vet Ther 4: 387-395

Český lékopis 01/2002: Vaccinum leptospirosis ad usum veterinarium: 0447

European Pharmacopoeia 01/2005: Canine leptospirosis vaccine (inactivated): 0447

Greenlee JJ, Bolin CA, Alt DP, Cheville, NF, Andreasen CB 2004: Clinical and pathological comparison of acute leptospirosis in dogs caused by two strains of Leptospira kirschneri serovar grippotyphosa. Am J Vet Res 65 : 1100-1107

Greenlee JJ, Alt DP, Bolin CA, Zuerner RI, Andreasen CB 2005: Experimental canine leptospirosis caused by Leptospira interrogans serovar pomona and Bratislava. Am J Vet Res 66: 1816-1822

Haake DA 2006: Hamster model of leptospirosis. Curr Protoc Microbiol Chapter 12: Unit 12E.2.

Klaasen HL, Molkenboer MJ, Vrijenhoek MP, Kaashoek MJ 2003: Duration of immunity in dogs vaccinated against leptospirosis with a bivalent inactivated vaccine. Vet Microbiol 95: 121-132

Koizumi N, Watanabe H 2005: Leptospirosis vaccines: Past, present, and future. J Postgrad Med 51: 210-214

Levett PN 2001: Leptospirosis. Clin Microbiol Rev 14: 296-326

Randall R, Cooper KH 1944: The goolden hamster (Cricetus auratus) as a test animal for the diagnosis of leptospirosis. Science 100: 133-134

Reed LJ, Muench HA 1938: Simple method of determining fifty percent endpoints. Am J Hyg 27: 494-497

Reed NE, Varney WC, Goddard RD, Wyeth PJ 2000: The maintenance of challenge strains used in the potency test for canine leptospira vaccines. Biologicals 28: 25-28

Roca B 2006: Leptospirosis. Rev Med Univ Navarra 50: 3-6

Schreiber P, Martin V, Najbar W, Sanquer A, Gueguen S, Lebreux B 2005: Prevention of a severe disease by a leptospira vaccination with a multivalent vaccine. Revue Méd Vét 156, 8-9: 427-432

Schreiber P, Martin V, Najbar W, Sanguer A, Gueguen S, Lebreux B 2005: Prevention of renal infection and urinary shedding in dogs by a leptospira vaccination. Vet Microbiol 108: 113-118

Silva EF, Santos CS, Athanazio DA, Seyffert N, Seixas FK, Cerqueira GM, Fagundes MQ, Brod CS, Reis MG, Dellagostin OA, Ko AI 2008: Characterization of virulence of Leptospira isolates in a hamster model. Vaccine. 26: 3892-3996. 
Srivastava SK 2006: Prospects of developing leptospiral vaccines for animals. Indian J Med Microbiol 24: 331 336

Vijayachari P, Sugunan AP, Shriram AN 2008: Leptospirosis: an emerging global public health problem. J Biosci 33: $557-569$

Vinetz JM 2001: Leptospirosis. Curr Opin Infect Dis 14: 527-538

Wang Z, Jin L, Wegrzyn A 2007: Leptospirosis vaccines. Microb Cell Fact 6: 39 